\title{
Simulation of the ion-induced shock waves effects on the transport of chemically reactive species in ion tracks ${ }^{\star}$
}

\author{
Pablo de Vera ${ }^{1,2,3, a}$, Eugene Surdutovich ${ }^{4}$, Nigel J. Mason ${ }^{2}$, Fred J. Currell ${ }^{1}$, and \\ Andrey V. Solov'yov ${ }^{2, b}$ \\ ${ }^{1}$ School of Mathematics and Physics, Queen's University Belfast, BT7 1NN Belfast, UK \\ ${ }^{2}$ Department of Physical Sciences, The Open University, Walton Hall, MK7 6AA Milton Keynes, UK \\ ${ }^{3}$ MBN Research Center, Altenhöferallee 3, 60438 Frankfurt am Main, Germany \\ ${ }^{4}$ Departament of Physics, Oakland University, Rochester, MI-48309, USA
}

Received 11 April 2018 / Received in final form 9 July 2018

Published online 7 September 2018

(C) The Author(s) 2018. This article is published with open access at Springerlink.com

\begin{abstract}
The passage of energetic ions through tissue initiates a series of physico-chemical events which leads to biodamage. The study of such ion-induced biodamage using a multiscale approach to the physics of radiation damage with ions has led to the prediction of shock waves being initiated by concentrated energy deposition along the ion track. In this work the radial energy deposition around carbon ion paths, calculated solving diffusion equations, is augmented with the inclusion of more energetic $\delta$-electrons. The radial profiles of energy deposition and the induced concentrations of free radicals are used to simulate the shock waves by means of reactive classical molecular dynamics, which predict a characteristic distribution of reactive chemical species which may have an as yet unrecognised contribution to the nascent biodamage. The paper also suggests some experimental methods by which such a shock wave may be detected and the predictions of these simulations verified.
\end{abstract}

\section{Introduction}

A basic understanding of the physico-chemical processes initiated by energetic ions in biomaterials is very important both for radiation protection and radiotherapy. In the former category, human beings are exposed to ion beams both from natural or human-made sources (e.g. cosmic radiation during manned space travel, Earth's natural radioactivity or workers exposure at nuclear reactors) [1]. In radiotherapy proton and heavier ion beams have been exploited since the 1990s in an advanced technique referred to as ion-beam cancer therapy (IBCT) [2-5]. Physical and chemical interactions initiated in biomaterials by the effect of ion radiation induce biological consequences in the irradiated cells and therefore it is essential to understand such interactions in order to improve on the radiation protection and optimization of therapy.

From a macroscopic point of view ion beams produce a depth-dose curve where the maximum of energy loss (the so-called Bragg peak) is reached close to the end of

\footnotetext{
* Contribution to the Topical Issue "Atomic Cluster Collisions", edited Alexey Verkhovtsev, Andrey V. Solov'yov, Germán Rojas-Lorenzo, and Jesús Rubayo Soneira.

${ }^{a}$ e-mail: p.devera@qub.ac.uk

${ }^{\mathrm{b}}$ On leave from A. F. Ioffe Physical Technical Institute, 194021 St. Petersburg, Russia.
}

the ion trajectories. This allows precise energy delivery to the tumor region while sparing surrounding healthy tissues by carefully selecting the initial energy of the ion beam. Microscopic patterns of energy deposition around each ion path feature intense radial doses which steeply decrease on a nanometer scale. In the Bragg peak region secondary electrons, free radicals and other reactive species are produced in large numbers, leading to much higher (compared to photon radiation) concentrations of DNA lesions and the formation of multiply-damaged sites, hence increasing the probability of cell death or sterilization because of the suppressed capability of enzymes to repair such a complex damage.

A thorough understanding of radiation damage with ions is needed to develop reliable optimization of IBCT treatment planning. The relationship between the cell survival probability and the physical dose is non-trivial. In a common X-ray therapy a single physical parameter, the dose, is involved, but the biological diversity is so staggering that empirical components in all existing models are essential. A number of molecular models have been developed since the 1960s with an effort to mathematically explain the experimental dependence of cell survival probabilities on dose [6]. For example, the microdosimetric kinetic model (MKM) [7] is one of the most advanced approaches that predicts cell survival as a function of dose and linear energy transfer (LET). A popular local effect model (LEM) $[2,8]$ relates the 
radial dose to the cell survival using its relation to the dose for cells irradiated with X-rays. The track-structure community aspires to include all relevant processes, from ionization/excitation of the medium with ions, including production, diffusion and reaction of secondary species, up to the description of nuclear DNA damage using Monte Carlo simulations $[9,10]$.

Another alternative is a multiscale approach (MSA) $[4,5,11]$ that takes a radically different direction. Instead of starting with the observed cell survival probabilities, pertinent physical, chemical, and biological processes are analyzed combining a variety of temporal, spatial and energy scales. One important distinction from the trackstructure approach is the prediction of cylindrical shock waves initiated by each ion propagating in the medium as a result of the energy deposited by the ion [12]. The strength of these waves increases with LET, so they might have a substantial contribution to radiation biodamage around the Bragg peak region. It should be noted that, although the ion-induced shock waves have not yet been experimentally confirmed (except for some indirect evidence as commented below), the successful comparison of experimental cell survival curves as a function of radiation dose with the MSA, which included the shock waves in the scenario, for a variety of cell lines, values of LET, oxygen environments and cell repair conditions [13], is impressive.

The predicted shock waves are a consequence of localized and fast energy transfer from the ions to the medium. In the vicinity of the Bragg peak most of secondary electrons have low energies of $\sim 45 \mathrm{eV}[14,15]$. These electrons transport the energy lost by the ion less than $1.5 \mathrm{~nm}$ around its path in some tens of femtoseconds, and there is no other mechanism which could dissipate this energy deposited so quickly. Indeed the molecular diffusion mechanism is too slow and the production of more energetic electrons ( $\delta$-electrons, which could carry the energy further away) vanishes in the Bragg peak region $[4,16]$. Thus the development of high pressures inside a narrow cylinder around the ion track is expected. In reference [12] it was demonstrated that the ratio of pressure inside and outside this narrow cylinder is larger than $(\gamma+1) /(\gamma-1)$, with $\gamma=C_{\mathrm{P}} / C_{\mathrm{V}}=1.222,{ }^{1}$ fulfilling the conditions for a "strong cylindrical explosion" [17]. This creates a discontinuity in the initial conditions for pressure that starts propagating in the radial direction away from the ion path. This propagation also features discontinuities in density and collective flow velocity and is referred to as shock wave $[17,18]$.

The onset of shock waves (or blast waves) as a result of concentrated and sudden energy deposition has been studied both theoretically and experimentally since early 1940s [19-25]. Analytical solutions for spherical shock waves where derived independently by Taylor [21], Zel'dovich [19] (those findings were reported later because of their military relevance), Sedov [22], and von Neumann [23]. These solutions are comprehensively presented in references $[17,18]$.

\footnotetext{
${ }^{1}$ This value used in reference [12] corresponds to vibrationally excited water molecules and thus gives the most conservative estimate for pressure difference.
}

Following the derivation for the spherical case, the hydrodynamic equations for the evolution of cylindrical shock waves resulting from the energy deposition around ion paths in liquid water (relevant to the current work) were solved in reference [12]. Later on, several molecular dynamics simulations of the dynamics of liquid water following energy deposition in the vicinity of ion paths within one picosecond of ions' traverse, confirmed the expected behavior of the theoretically predicted shock waves [26-28].

The intial energy transfer from the ions to the medium, which sets on the initial conditions for the shock wave, happens $\sim 10^{-13} \mathrm{~s}$ after ion traversal, when all electrons have been thermalized. Thus the shock wave propagates on a time scale between the so-called physical and chemical stages of radiation, typically considered to be fairly well separated in time and both preceding the biological stage [29]. While the evolution of track structure finishes by $\sim 10^{-13} \mathrm{~s}$ (at the end of physical stage) and reactive chemical species (free radicals and solvated electrons) are created by $\sim 10^{-13}-10^{-12} \mathrm{~s}$, the chemical stage is only deemed to start $\sim 10^{-12} \mathrm{~s}$ after irradiation and last until $\sim 10^{-6}$ s. However, the emerging waves may have a substantial influence on the radiation chemistry, effectively mixing the physical and chemical stages.

Two possible effects of the ion-induced shock waves on the radiation damage scenario have been suggested [30]: the first is the direct DNA damage by thermo-mechanical stress; the second is their role in spreading reactive species out of the ion track. Classical molecular dynamics simulations were previously used to investigate the first aspect in the Bragg peak region for different ions $[26,27,30]$. It was shown that covalent bonds can be ruptured by stress if the target is close enough to the ion path and the LET is sufficiently large.

Regarding the second effect, in reference [30] it was also predicted that shock waves may play a significant role in the transport of reactive species (such as free radicals) due to radial collective flows initiated by them. The analysis of formation and diffusion of free radicals in reference [16] suggests that, if there are no shock waves, most of the radicals do not leave the ion track since they annihilate due to high rates of chemical reactions, high concentrations and the inability of the diffusion mechanism to steer them outside. Although this idea has been heavily exploited $[4,13]$ it has never been properly studied, hence the first quantitative analysis of this effect being the aim of this work.

To date the ion-induced shock waves (and their impact on the radiation chemistry) have not been observed directly. However, there is some evidence that makes their existence plausible. The detected acoustic waves from the Bragg peak region [31] are likely to be the artifacts of shock waves. Moreover the only water radiolysis experiment for very high-LET ions known to us revealed an unusually large number of radicals escaping the ion tracks [32], an effect that might be caused by their transport in the collective flow. In any case, as will be commented later on, the results of this work suggest some potential experiments that may be used to detect the signature of the shock waves on the ion-induced radiation chemistry. 
This article reports on molecular dynamics simulations of carbon ion-induced shock waves (carbon being one of the most promising ions used in therapy) in which their strength has been evaluated both in the Bragg peak region and out of it. Furthermore, a study of transport of the reactive hydroxyl radicals produced in the ion track by the induced cylindrical collective flow is performed and its impact on the initial stage of the radiation chemistry is evaluated, by comparison with simulations where the shock wave effect is disregarded.

First, the augmented initial distributions of energy deposition and radicals around the ion path are obtained by accounting for the effect of $\delta$-electrons in addition to those obtained by solving the diffusion equations for low energy electrons [16]. The effect of $\delta$-electrons is crucial for the description of track structure for high energy ions out of the Bragg peak region. Calculated radial doses are compared to Monte Carlo simulations. Classical molecular dynamics is used to simulate the shock wave, using the obtained radial doses to set up their initial conditions. We investigate the effect of the radial-dose including $\delta$-electrons on the strength of the shock wave both in and out of the Bragg peak region, and the results are interpreted in terms of the hydrodynamic equations [12]. Finally, the new reactive force field implemented in MBN Explorer [33,34] allows us to simulate one of the most representative chemical reactions occurring around the ion path (OH recombination to give hydrogen peroxide) in the presence of the wave. This allows us to study its influence on the initial stage of the radiation chemistry. To benchmark the reactive classical molecular dynamics simulations an additional simulation for low-LET protons is reported: in this case shock waves will not play an important role and thus our results for pure diffusion-reaction can be directly compared to simulations performed with a popular Monte Carlo package, GEANT4-DNA [35].

The paper is organized as follows: Section 2.1 describes the calculation of the radial doses and the initial distributions of $\mathrm{OH}$ radicals produced around energetic ion tracks by means of diffusion equations for low energy electrons and the consideration of energetic $\delta$-electrons. The radial doses are used to set up initial conditions for the shock wave simulation by classical molecular dynamics as it is explained in Section 2.2. The extension of the classical molecular dynamics procedure to include chemical reactivity, by using the reactive CHARMM force field implemented in MBN Explorer, is explained in Section 2.3. This allows us to simulate $\mathrm{OH}$ recombination in the presence of the shock wave by means of classical molecular dynamics. The results of the simulations are presented and analyzed in Section 3, and the final conclusions and remarks are given in Section 4.

\section{Methods}

\subsection{Calculation of the radial doses and the $\mathrm{OH}$ radical densities around energetic ion paths}

In references $[4,11,16]$ the transport of electrons in the vicinity of the Bragg peak was treated analytically using

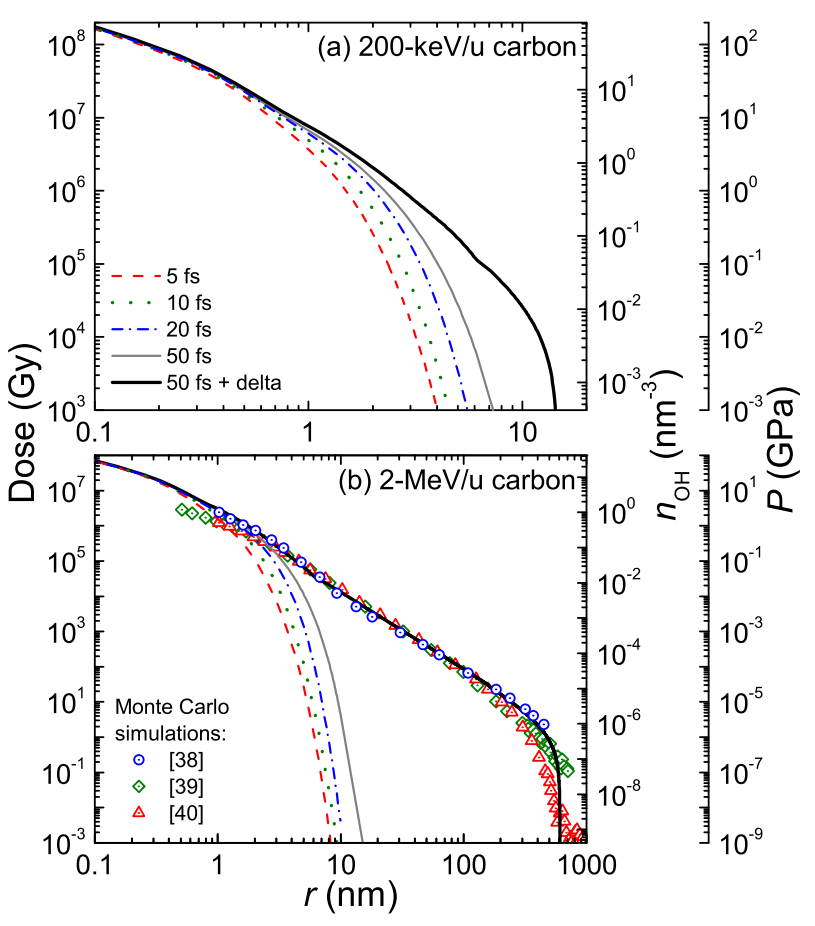

Fig. 1. Left axis: radial doses produced in liquid water by (a) $200 \mathrm{keV} / \mathrm{u}$ and (b) $2 \mathrm{MeV} / \mathrm{u}$ carbon ions. Thin lines represent the time evolution of radial dose calculated using the diffusion equations, while thick lines represent final radial dose distributions including the $\delta$-electrons contribution. Symbols correspond to Monte Carlo simulations results [38-40]. Right axes: radial pressure and $\mathrm{OH}$ radical distributions produced by ions after $\sim 0.1 \mathrm{ps}$ (see the text for details).

the diffusion equations and the radial dose deposition profile at a given time was calculated [16]. This approach is justified in the Bragg peak region since most of the ejected electrons there have kinetic energies below $50 \mathrm{eV}$ $[4,14,15]$ and their propagation can safely be described by successive elastic collisions which randomly change their direction of motion. These low energy electrons have enough energy for producing one more ionization of water molecules. This brings about a second generation of lower energy electrons which then thermalize after the next inelastic event. The diffusion equations for two generations of low energy electrons were solved in reference [16].

The diffusion equation approach [16] was developed for the Bragg peak region and describes the transport of only low-energy electrons. Higher energy $\delta$-electrons (which are kinematically constrained in the Bragg peak region) have to be accounted for in order to describe the transport in the ion track proximal to the Bragg peak. $\delta$-electrons are responsible for the formation of the tail in the radial dose distribution at large radii (see Monte Carlo results for $2 \mathrm{MeV} / \mathrm{u}$ carbon ions in water in Fig. 1). In order to simulate the effect of shock waves out of the Bragg peak in this work we have implemented a recipe to account for $\delta$-electrons based on a spatially-restricted LET formula [36] while the low-energy electrons are still treated by diffusion equations. The technical implementation goes beyond the goal of this paper and is described in another work [28]. 
In Figure 1 the time evolution of the radial dose produced by a carbon ion (a) in the Bragg peak region (energy $200 \mathrm{keV} / \mathrm{u}$ ) and (b) at $2 \mathrm{MeV} / \mathrm{u}$ (out of the Bragg peak) calculated by the diffusion equations is shown by thin lines. The radial dose increases with time until it saturates at $\sim 50 \mathrm{fs}$, when all electrons have thermalized. It should be noted that there is no mechanism by which energy deposited so quickly can be dissipated gradually, since processes such as electron-phonon interaction or diffusion take place on longer time scales [16,37]. The radial doses corresponding to the final stage of the track-structure, accounting for $\delta$-electrons, are represented in Figure 1 by thick lines and compared to different Monte Carlo simulations for $2 \mathrm{MeV} / \mathrm{u}$ carbon ions [38-40]. The present calculations are in rather good agreement with the Monte Carlo results demonstrating the capacity of this approach to correctly predict the radial doses. Our calculations correspond to the final stage of the track structure but before the shock wave is created and this is why they agree with the Monte Carlo simulations where shock waves have not yet been included.

Interestingly the same profile of the radial dose gives an upper estimate for the pressure developed around the ion path. As shown in reference [16], the radial dose is equal to the pressure profile if the energy deposited into the electronic system of the target has been transferred to the nuclear system through electron-phonon coupling. Estimates based on the thermal-spike model indicate that this happens approximately by $\sim 0.1 \mathrm{ps}$ [41]. Thus, the pressure profiles around carbon ion tracks by $\sim 0.1$ ps after ion traversal are shown in Figure 1 (the axis is given on the right). Such large pressures are sufficient to initiate shock waves in the liquid medium. The hydrodynamic equations with the initial conditions corresponding to a strong explosion were solved in reference [12]. This solution allows one to benchmark the wave front characteristics obtained by molecular dynamics simulations, as it was shown in reference [27], so we will make use of it later on in Section 3.

Besides the dose and pressure profiles the diffusion equations also yield the initial distribution of free radicals and pre-solvated electrons [16]. The radicals are the product of chemical evolution of $\mathrm{H}_{2} \mathrm{O}^{+}$ions and excited water molecules $\mathrm{H}_{2} \mathrm{O}^{*}$ formed in the processes of primary and secondary ionization and excitation events $[16,47]$. In the first approximation it can be assumed that each inelastic collision leads to the formation of one $\mathrm{OH}$ radical by dissociation of a water molecule:

$$
\begin{gathered}
\mathrm{H}_{2} \mathrm{O}^{+} \longrightarrow \cdot \mathrm{OH}+\mathrm{H}^{+}, \\
\mathrm{H}_{2} \mathrm{O}^{*} \longrightarrow \cdot \mathrm{OH}+\cdot \mathrm{H} .
\end{gathered}
$$

Under this assumption the initial distribution of $\mathrm{OH}$ will follow the profile of the radial dose [16] as shown in Figure 1. Proton transfer in the ionized water molecule dissociation, as well as proton and electron solvation, are fast procesess occurring within $\sim 10^{-14}-10^{-13} \mathrm{~s}[29]$. Therefore, by the time that the shock wave develops $\left(\sim 10^{-13} \mathrm{~s}[12,16,41]\right)$, the radicals are almost at the same location where they were created and most of the energy transferred to electronically excited water molecules is released to the medium as thermal energy. This approach for estimating the production of $\mathrm{OH}$ radicals, to be used later on for molecular dynamics simulations, is similar to that recently presented in the works by Abolfath et al. $[42,43]$.

\subsection{Molecular dynamics simulation of the shock waves}

The dynamics of the medium can be conveniently simulated by means of classical molecular dynamics, this technique having been previously used to assess the effects of ion-induced shock waves in biomaterials $[27,30]$. In molecular dynamics simulations [44] the classical trajectories of all the atoms of the system, determined by their mutual interaction forces, are followed in time. The coordinates and velocities of each atom are found at discrete time steps by numerically solving their Langevin equations $[27,33]$ where both their interaction forces with all the rest of atoms in the system and the energy exchange with a heat bath at temperature $\mathcal{T}$ are accounted for. In biological systems and liquid water the interaction forces between groups of atoms can be conveniently described by the use of empirical force fields among which one of the most popular is CHARMM [45]. In CHARMM the potential energy of the vector of atomic coordinates $\boldsymbol{R}$ is evaluated as:

$$
\begin{aligned}
U(\boldsymbol{R})= & \sum_{\text {bonds }} U_{i j}^{\text {bond }}\left(r_{i j}\right)+\sum_{\text {angles }} U_{i j k}^{\text {angle }}\left(\theta_{i j k}\right) \\
& +\sum_{\text {dihedr. }} U_{i j k l}^{\text {diedr. }}\left(\chi_{i j k l}\right)+\sum_{\text {improper }} U_{i j k l}^{\text {improper }}\left(\phi_{i j k l}\right) \\
& +\sum_{i} \sum_{j \neq i}\left\{\frac{q_{i} q_{j}}{\varepsilon r_{i j}}+\epsilon_{i j}\left[\left(\frac{R_{\min , i j}}{r_{i j}}\right)^{12}-\left(\frac{R_{\min , i j}}{r_{i j}}\right)^{6}\right]\right\} .
\end{aligned}
$$

The terms in the first two lines of the right hand side of this equation correspond to the sum of all the bonding interactions, while the last two are non-bonding interactions. The bonding interactions correspond to the potential energies arising from the distance $r_{i j}$ between two bound atoms $i$ and $j$, the angle $\theta_{i j k}$ between three sequentially bound atoms $i, j$, and $k$, and the dihedral and improper angles, $\chi_{i j k l}$ and $\phi_{i j k l}$, formed by groups of four sequentially bound atoms $i, j, k$ and $l$, and their deviation from their equilibrium values. The nonbonding interactions are given by the Coulomb and the van der Waals interactions, the latter represented here by a Lennard-Jones potential of well depth $\epsilon_{i j}$ and distance at the potential minimum $R_{\min , i j} . q_{i}$ are the partial charges of the atoms $i$ and $\varepsilon$ is the effective dielectric constant of the system. Parameters for water and biological macromolecules are given by CHARMM [45]. The particles that interact through bonding or non-bonding interactions are determined by the topology of the system, which defines the connectivity of the atoms. It should be noted that, in the standard CHARMM force field, this topology is fixed and cannot vary during the simulation.

In the standard CHARMM force field all the bonding interactions are described by harmonic potentials. For example the first term in the right hand side of 
equation (2) is given by:

$$
U_{i j}^{\text {bond }}\left(r_{i j}\right)=k_{i j}^{\text {bond }}\left(r_{i j}-r_{0, i j}\right)^{2},
$$

where $k_{i j}^{\text {bond }}$ is the force constant of the bond between the atoms $i$ and $j, r_{i j}$ is their relative distance and $r_{0, i j}$ is their equilibrium distance. Similar expressions are given for the rest of bonding interactions [45]. This allows us to describe the atoms motion around their equilibrium configuration but it does not allow bond breaking (or formation). In order to account for chemical reactivity an extension of this force field will be used in this work, which is described in the next subsection.

The radial doses calculated in Section 2.1 and shown in Figure 1 can be used to set up initial velocities for each atom $i$ for the molecular dynamics simulation of liquid water response, $v_{i}^{\mathrm{MD}}$, which might be in the form of a shock wave if the energy deposited is concentrated enough. Simulations are arranged as described in reference [27]: liquid water simulation boxes are constructed and preequilibrated at body temperature $\mathcal{T}=310 \mathrm{~K}$, after which equilibrium velocities $v_{i}^{\text {eq }}$ are obtained. These velocities are then used to get $v_{i}^{\mathrm{MD}}$ according to the energy deposited around the ion track. When there is no shock wave, $v_{i}^{\mathrm{MD}}=v_{i}^{\mathrm{eq}}$; when there is a shock wave, $v_{i}^{\mathrm{MD}}=v_{i}^{\text {neq }}$, where the non-equilibrium velocities $v_{i}^{\text {neq }}$ are obtained as described below.

In previous works it was assumed that the radial doses (in the Bragg peak region) are so steep that all the energy lost by the ion is deposited in a cylinder of radius $1 \mathrm{~nm}$, the so called "hot cylinder", and atomic velocities of the atoms after equilibration $\left(v_{i}^{\mathrm{eq}}\right)$ were scaled only inside this cylinder $[26,28,30]$. In this work we account for the spreading of the radial dose in a more accurate way and the atomic velocities are scaled as follows. The ion path is considered to cross the center of the simulation box in the $y$ direction. The box may then be divided in concentric cylindrical shells of water molecules of thickness $\mathrm{d} r=1 \AA$ around the ion path. The velocities at equilibrium $v_{i, j}^{\text {eq }}$ of atoms $i$ (of mass $m_{i, j}$ ) at each shell $j$ can be scaled ${ }^{2}$ by a parameter $\alpha_{j}$ depending on the amount of energy deposited in this shell, to give the initial velocities for the simulations $v_{i, j}^{\mathrm{MD}}=v_{i, j}^{\text {neq }}=\alpha_{j} \cdot v_{i, j}^{\text {eq }}$. The kinetic energy of the shell $j$ after energy deposition by the ion is then given by:

$$
\begin{aligned}
\sum_{i}^{N_{j}} \frac{1}{2} m_{i, j}\left(v_{i, j}^{\mathrm{neq}}\right)^{2} & =\sum_{i}^{N_{j}} \frac{1}{2} m_{i, j}\left(\alpha_{j} \cdot v_{i, j}^{\mathrm{eq}}\right)^{2} \\
& =\frac{3 N_{j} k_{\mathrm{B}} \mathcal{T}}{2}+f\left(r_{j}\right) S L,
\end{aligned}
$$

and this equation is used to determine $\alpha_{j}$. The first term on the right hand side of the equation corresponds to the initial kinetic energy of the atoms in the cylindrical shell $j$ (with $N_{j}$ atoms) at equilibrium $(\mathcal{T}=310 \mathrm{~K}), k_{\mathrm{B}}$ being the Boltzmann's constant. The second term is the energy deposited by the ion in the shell $j$ when crossing the

\footnotetext{
${ }^{2}$ Note that only the moduli of the velocities are scaled, while their directions are unaltered; thus, the total momentum is conserved, and the radial collective flow arises naturally.
}

system which is its stopping power $S$ (the average energy lost by the ion per unit path length) times the length of the simulation box $L$ times the fraction of the energy deposited in this shell $f\left(r_{j}\right)$ of inner radius $r_{j}$ and width $\mathrm{d} r$. This fraction is obtained from the radial dose $d(r)$ as:

$$
f\left(r_{j}\right)=\frac{2 \pi r_{j} \mathrm{~d} r L \rho d\left(r_{j}\right)}{\sum_{k=1}^{\infty} 2 \pi r_{k} \mathrm{~d} r L \rho d\left(r_{k}\right)}=\frac{2 \pi r_{j} \mathrm{~d} r \rho d\left(r_{j}\right)}{S}
$$

where $\rho$ is the mass density of liquid water.

It should be noted that in molecular dynamics simulations the Langevin thermostat is only used during the preequilibration of the system at temperature $\mathcal{T}$, while it is switched off during the shock wave simulations in order to conserve the energy deposited by the ion. A small fraction of this energy may be subtracted from the right hand side of equation (4) due to the residual energy stored in the final products of water molecules dissociation in the liquid environment. However, as discussed at the end of Section 2.1, it is expected that almost all of this energy is quickly liberated to the medium again, so we do not account for this effect in the present simulations. Further refinements in this direction will be considered in future work.

\subsection{Reactivity of $\mathrm{OH}$ radicals: the reactive CHARMM force field}

Chemical reactivity can be simulated within molecular dynamics using the new reactive CHARMM force field introduced and implemented in MBN Explorer [34]. This extension allows us to include chemistry merely by defining a few additional parameters, hence introducing reactivity without a significant increase in the computational cost.

In the reactive force field, the harmonic bond interaction described by equation (3) is replaced by a Morse potential of the form:

$$
U_{i j}^{\text {bond }}\left(r_{i j}\right)=D_{i j}\left[e^{-2 \beta_{i j}\left(r_{i j}-r_{0, i j}\right)}-2 e^{-\beta_{i j}\left(r_{i j}-r_{0, i j}\right)}\right] .
$$

Here $D_{i j}$ represents the dissociation energy for the bond between the atoms $i$ and $j$. The parameter $\beta_{i j}$ determines the steepness of the potential and it can be demonstrated that it is given by the relation $\beta_{i j}=\sqrt{k_{i j}^{\text {bond }} / D_{i j}}$ [34]. An additional parameter can be defined, the cutoff distance, characterizing the distance beyond which the bond can be considered to be broken (or below which a new bond is considered to be formed). Within the Morse potential description the potential energy of the bond does not grow indefinetely as $r_{i j}$ increases but it approaches zero until the bond is broken. Accordingly, switching functions are defined to gradually reduce the angular and dihedral interactions as the bond breaks [34].

As a first approximation for the induced radiation chemistry, in this work only one of the most relevant reactions is considered, $\mathrm{OH}$ recombination to give hydrogen peroxide:

$$
\cdot \mathrm{OH}+\cdot \mathrm{OH} \longrightarrow \mathrm{HO}-\mathrm{OH},
$$




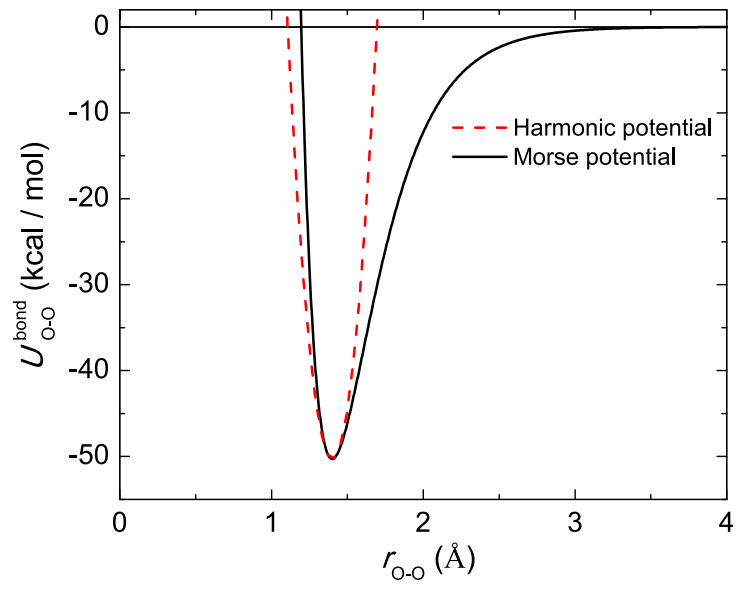

Fig. 2. Potential energy for the hydrogen peroxide $\mathrm{O}-\mathrm{O}$ bond in the standard CHARMM force field (dashed line) and in the reactive force field (solid line).

since $\mathrm{OH}$ is the most important species in chemical biodamage [46]. For simplicity in this work we disregarded any other chemical reactions [47], so pre-solvated electrons and hydrogen radicals were not included in the simulations. OH radicals are produced in water by the fragmentation of excited water molecules (Eq. (1)) and their radial distribution follows the radial dose, as explained in Section 2.1. To assure charge neutrality protons coming from the water dissociation were removed from the system. A similar approach, where $\mathrm{OH}$ reaction with DNA molecules in water following ionizing radiation was included, has been reported recently [42,43]. However, in these works shock waves were not considered, and here we do not include DNA molecules, in order to understand first the role of shock waves in the pure liquid water medium. Interaction of shock waves, $\mathrm{OH}$ radicals and DNA will be considered in future work.

Since $\mathrm{O}-\mathrm{H}$ bonds are stronger than $\mathrm{O}-\mathrm{O}$ bonds in peroxide $[48,49]$ in our simulations these bonds were assumed to be non-reactive while the only possible reaction considered is the formation and breaking of the $\mathrm{O}-\mathrm{O}$ bond. The parameters needed are the $\mathrm{O}-\mathrm{H}$ and $\mathrm{O}-\mathrm{O}$ bond distances and force constants, the $\mathrm{O}-$ $\mathrm{O}$ bond dissociation energy and cutoff distance, the atomic partial charges in the $\mathrm{OH}$ radical and peroxide molecule and the equilibrium angle and force constant for the O-O-H angle [34]. Typical values for these parameters have been taken from the literature: the $\mathrm{O}-\mathrm{H}$ distances are similar as those in water [50] so CHARMM parameters have been kept $\left(r_{0, \mathrm{OH}}=0.9572 \AA\right.$ and $\left.k_{\mathrm{OH}}^{\text {bond }}=450 \mathrm{kcal} / \mathrm{mol} / \AA^{2}\right)[45]$. The values for the O-O bond and the $\mathrm{O}-\mathrm{O}-\mathrm{H}$ angle have been taken from reference $[51], r_{0, \mathrm{OO}}=1.453 \AA, k_{\mathrm{OO}}^{\text {bond }}=540 \mathrm{kcal} / \mathrm{mol} / \AA^{2}$, $\theta_{0, \mathrm{OOH}}=102.7^{\circ}$, and $k_{\mathrm{OOH}}^{\text {angle }}=140 \mathrm{kcal} / \mathrm{mol} / \mathrm{rad}^{2}$. The dissociation energy of the $\mathrm{O}-\mathrm{O}$ bond for peroxide in the gas phase $D_{\mathrm{OO}}=50.29 \mathrm{kcal} / \mathrm{mol}$ has been taken from experimental data [52]. Partial charges of $\pm 0.375 e$ have been used for $\mathrm{OH}[50]$ and $\pm 0.35 e$ for peroxide [51]. Inspection of the resulting $\mathrm{O}-\mathrm{O}$ Morse potential, shown in Figure 2, gives a cutoff distance of $\sim 3 \AA$. As it can be seen in the figure, the Morse potential resembles the standard harmonic potential used in CHARMM around the equilibrium bond distance. However, as the bond distance increases the potential monotonically goes to zero until the bond is effectively broken for distances larger than $\sim 3 \AA$.

The performance of this reactive force field has been previously demonstrated in a description of water dissociation at high temperatures [34] and it will be further checked for the present case in Section 3.

\section{Results and discussion}

In this section we will first analyze the strength of the carbon ion-induced shock waves in the Bragg peak region and out of it. The effect of the characteristic radial doses (where the role of $\delta$-electrons is much more important at high energies) in the shock wave simulations is analyzed in Section 3.1 and interpreted in terms of the hydrodynamic model predictions [12]. We then analyze the time evolution of the radiation chemistry and the effect on it of the shock waves for a high- and low-LET cases in Section 3.2.

\subsection{Strength of the ion-induced shock wave in and out of the Bragg peak region}

First let us analyze the general characteristics of the carbon ion-induced shock waves for the two energies for which radial doses have been presented in Figure 1: $200 \mathrm{keV} / \mathrm{u}$ (energy characteristic of the Bragg peak region) and $2 \mathrm{MeV} / \mathrm{u}$ (a larger energy found out of the Bragg peak region). The LET for the former case is $112.5 \mathrm{eV} / \AA$ and for the latter $56.53 \mathrm{eV} / \AA$. Furthermore, the radial doses are significantly different: in the Bragg peak the contribution to the dose of energetic $\delta$-electrons is very small, while they transport the energy further away in the latter case, since more energetic electrons are produced by high energy ions. In the following we will analyze the impact of the radial doses on the pressure of the wave front of the generated shock waves and we will compare the results with previous work where the "hot cylinder" approximation (i.e., step-function-distributed initial pressure) was used for the Bragg peak region [27]. Molecular dynamics results will also be analyzed in terms of the hydrodynamic equations solved in reference [12].

The dependencies of pressure at the wave front obtained from molecular dynamics simulations for carbon ions in and out of the Bragg peak region on the radius of the wave front, obtained as explained in reference [27], are shown in Figure 3. The insets show the evolution of radii of wave fronts as a function of time. The results for the stepfunction-distributed initial pressure (hot cylinder, circles) are compared to those for the initial pressure distributed in accordance with the radial dose (squares). The hydrodynamic calculations for the cylindrical strong explosion case (dashed lines) [12] agree with the hot cylinder simulations, confirming that a shock wave is observed in the simulations [27,28]. However, the use of the radial dose reduces the intensity of the wave front. This reduction is up to $30 \%$ for a given radius in the case of the Bragg 


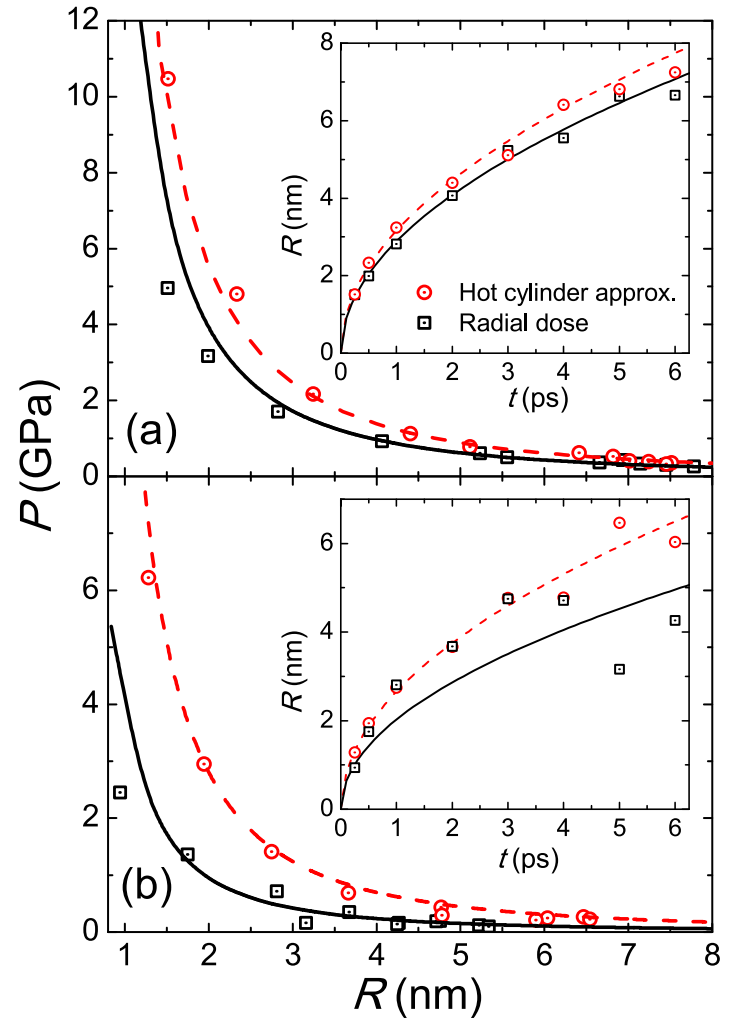

Fig. 3. Pressure at the wave front as a function of its position for the shock wave produced by (a) $200 \mathrm{keV} / \mathrm{u}$ and (b) $2 \mathrm{MeV} / \mathrm{u}$ carbon ions. The insets depict the wave front position as a function of time. Symbols represent simulation results, while lines correspond to the hydrodynamic model.

peak region (for which the hot cylinder model has been applied previously $[26,27,30])$, mainly due to the dispersion of the initial wave front, but it reaches stupendous $65 \%$ (for a given radius) for $2 \mathrm{MeV} / \mathrm{u}$ carbon ions where $\delta$-electrons become important. For the more realistic simulations using the radial dose the hydrodynamic model also reproduces the simulation results (solid lines in the figure) but assuming a reduced "effective" stopping power. The latter corresponds (for both energies) to the amount of energy deposited within the first $\sim 1-2 \mathrm{~nm}$ from the ion path [28]. While in the Bragg peak region most of the energy is deposited within this cylinder this is not the case at higher energies outside the Bragg peak due to the production of more energetic $\delta$-electrons.

Therefore, the first conclusions are that (i) ion-induced shock waves are observed in the simulations (for both energies) as it is confirmed by the agreement with the hydrodynamic equations, and they initiate a cylindrical collective flow; (ii) the shock waves/collective flows will be strong for the high LET and concentrated radial doses characteristic of the Bragg peak region (where the "hot cylinder" approximation previously used $[26,27,30]$ works reasonably well), while they are strongly damped for the higher energies out of the Bragg peak region. This damping is due to a larger spread of radial dose due to $\delta$-electrons resulting in the reduction of pressure gradients.

\subsection{Time evolution of the radiation chemistry for different LET}

Once the strength of the shock waves for different LET has been determined it is possible to explore their effects on the chemical environment produced by the ion tracks. The ion-induced shock waves (and the subsequent collective flows) are not considered in current biophysical models, with the exception of the multiscale approach (MSA) [13], so the aim of this section is to determine which effects are being missed when the shock waves are not included in the modeling. This aspect can be easily studied by simulation, where the shock wave can be artificially "switched off" (by setting the initial atomic velocities to those at equilibrium, $\left.v_{i, j}^{\mathrm{MD}}=v_{i, j}^{\mathrm{eq}}\right)$.

As described above, the diffusion equations also give the initial distribution of $\mathrm{OH}$ radicals around the ion path, as shown in Figure 1. This distribution can be used as the initial condition for molecular dynamics simulations in which $\mathrm{OH}$ transport and reaction can be included. In principle, reaction of $\mathrm{OH}$ radicals with DNA molecules can also be included in the simulations, as done, e.g., in references $[42,43]$. However, this is beyond the scope of the present work, where we will restrict ourselves to study the effects of shock waves on the radiation chemistry induced in pure water. Results for two limiting cases will be shown: (i) carbon ions in the Bragg peak $(200 \mathrm{keV} / \mathrm{u}, \mathrm{LET}=112.5 \mathrm{eV} / \AA)$, where the effects of the shock waves are expected to be stronger according to the results of the previous subsection, and (ii) a case of low LET $(4.1 \mathrm{eV} / \AA)$ for $500 \mathrm{keV}$ protons. We have chosen the latter case because the LET is roughly thirty times smaller than for carbon in the Bragg peak, so we can safely assume that there will not be a shock wave (or it will be very weak). For this case, our simulations for the evolution of the chemistry can be compared with other calculations reported using the GEANT4-DNA package, which implements the well-known diffusion-reaction algorithms [35].

For performing these simulations, we have converted the $\mathrm{OH}$ concentration $n_{\mathrm{OH}}(r)$ (Fig. 1) into a histogram of $5 \AA$ bin width, and the appropriate number of water molecules from the simulation box have been randomly selected and deprotonated in each bin, leaving neutral $\mathrm{OH}$ radicals randomly distributed inside the bin but following the calculated radical density. The results of this procedure for the two described cases are shown in Figure 4, where two samples of the generated initial distributions of $\mathrm{OH}$ radicals around the ions paths are depicted. These correspond to the initial conditions of simulations in which the transport and reactivity of $\mathrm{OH}$ radicals are studied. It should be noted how the big differences in LET are manifested in the production of $\mathrm{OH}$ radicals: while $500 \mathrm{keV}$ protons produce a sparce concentration of radicals along the trajectory, $200 \mathrm{keV} / \mathrm{u}$ carbon ions produce large densities of them, which will increase their chance to recombine. To have a significant number of radicals in each simulation box, the length of the water box in case (i) was chosen to be just $43 \AA$ (132 radicals), while in case (ii) we had to increase it to $305 \AA$ (52 radicals). 


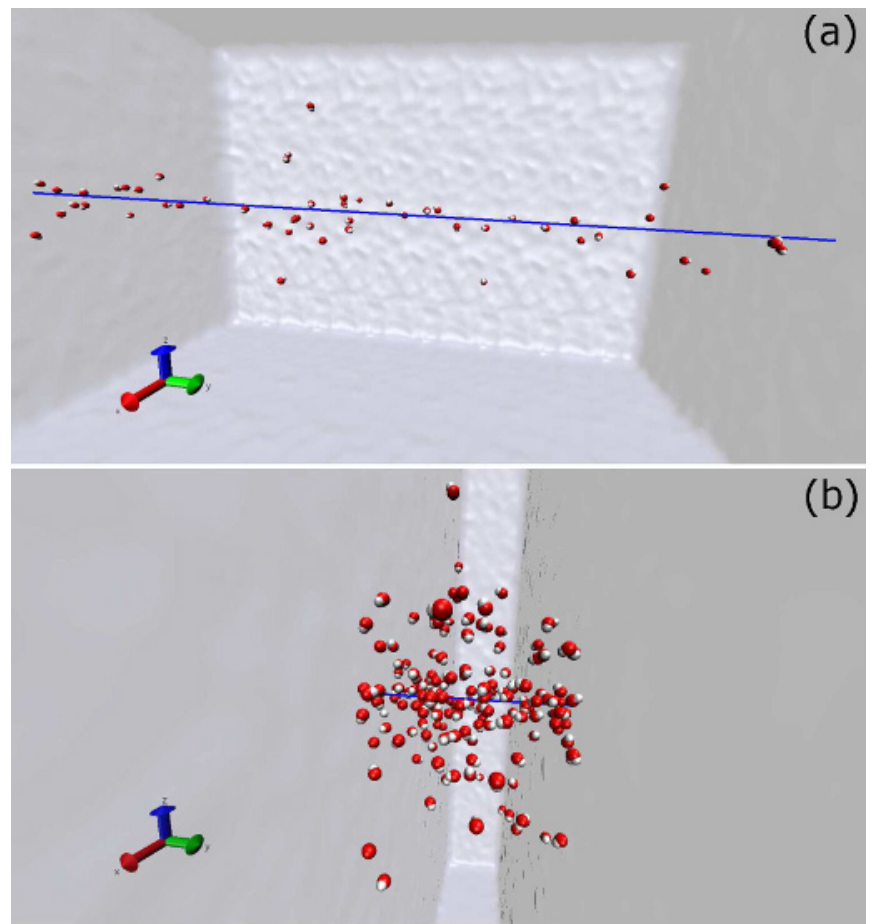

Fig. 4. $\mathrm{OH}$ radical distributions produced in liquid water around (a) a $500 \mathrm{keV}$ proton path and (b) a $200 \mathrm{keV} / \mathrm{u}$ carbon ion path. The solid line represents the ion path, while spheres represent the $\mathrm{OH}$ radical atoms ( $\mathrm{red}=$ oxygen, white $=$ hydrogen), and the rest of water molecules in the environment are not displayed for clarity. See the text for details.

Let us start the analysis by studying the case of $500 \mathrm{keV}$ protons: in this low LET case $(4.1 \mathrm{eV} / \AA$, as compared to $112.5 \mathrm{eV} / \AA$ for carbon in the Bragg peak region) we do not expect a shock wave to occur. Therefore we simulate the diffusion of chemically active $\mathrm{OH}$ radicals. The results can be directly compared to the GEANT4-DNA results reported in reference [35], what allows us to benchmark the choice of the model parameters for the reactive force field described in Section 2.3. Figure 5a depicts by symbols the results for the mean square displacement MSD of the $\mathrm{OH}$ radicals in a simulation where chemistry is not allowed. This permits us to obtain the diffusion coeffitient $D_{\mathrm{OH}}$ in a pure diffusive situation. According to the Einstein equation, MSD $=6 D_{\mathrm{OH}} t$, so from the slope of the line fitting the results we get $D_{\mathrm{OH}}=0.22 \AA^{2} / \mathrm{ps}$. This is in a relatively good agreement with the results of recent simulations giving $0.3 \AA^{2} / \mathrm{ps}$ [50] and with the value used in the popular simulation packages PARTRAC [53] or GEANT4-DNA [54], $0.28 \AA^{2} / \mathrm{ps}$, so pure diffusion is reasonably well simulated.

Figure 5b shows by an histogram the evolution of the G-value for $\mathrm{OH}$ radicals (number of molecules produced per $100 \mathrm{eV}$ of deposited energy). The histogram shows the average of three independent simulations (in which different random distributions of free radicals have been generated following the radial dose distribution) where the error bars represent the standard deviation. MBN Explorer results are compared to the results provided by

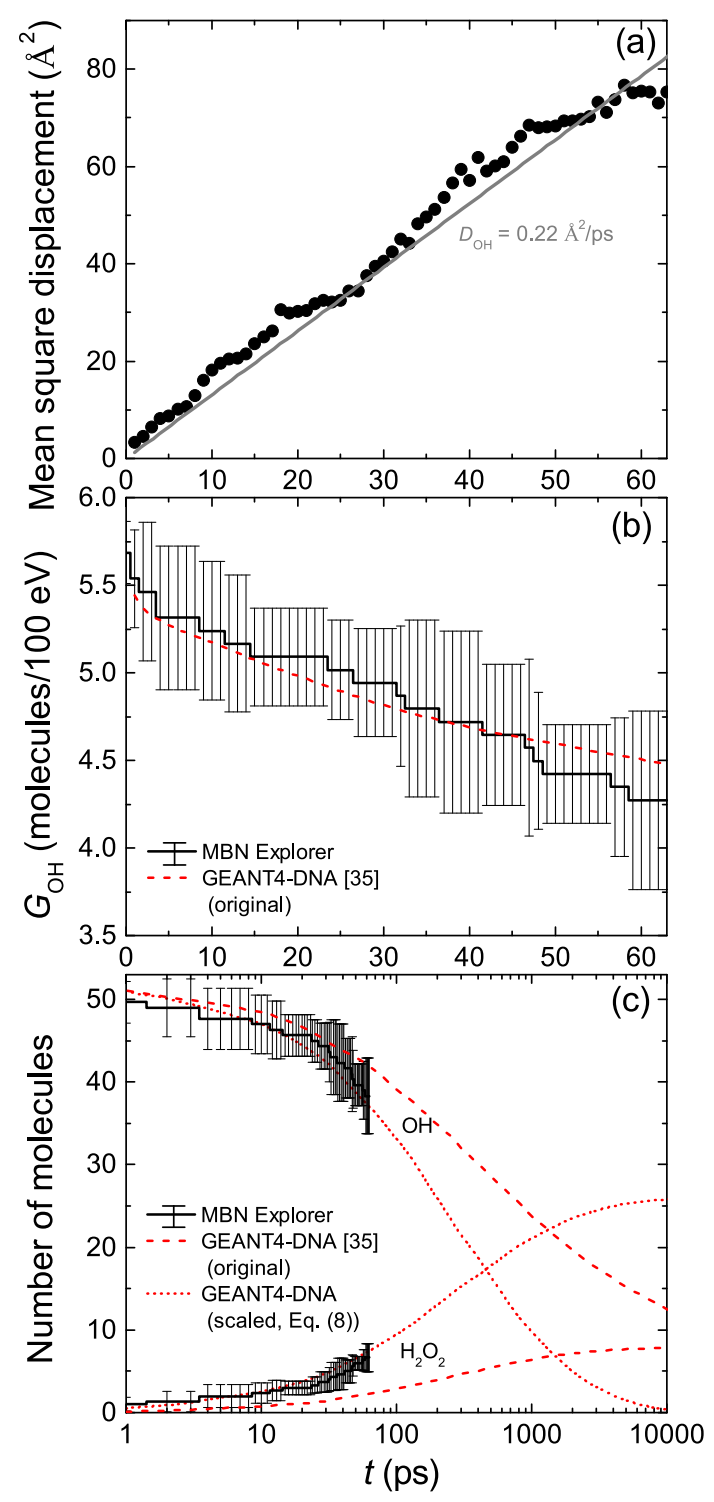

Fig. 5. Diffusion and reactivity of the $\mathrm{OH}$ radicals produced by $500 \mathrm{keV}$ protons in liquid water, where a shock wave is not expected to occur due to the very low LET. (a) Mean square displacement of $\mathrm{OH}$ radicals, (b) $\mathrm{G}$-value for $\mathrm{OH}$ radicals, and (c) number of $\mathrm{OH}$ and $\mathrm{H}_{2} \mathrm{O}_{2}$ molecules, as a function of time. MBN Explorer results (histograms with error bars) are compared to GEANT4-DNA simulations (dashed and dotted lines) [35]. See the text for details.

GEANT4-DNA (dashed line) [35]. The agreement is excellent, even having into account the fact that in this work we only consider the recombination reaction (7), while in GEANT4-DNA many more reaction channels (several of them involving $\mathrm{OH}$ ) are included.

These results are further compared in Figure $5 \mathrm{c}$, where we show the number of $\mathrm{OH}$ and $\mathrm{H}_{2} \mathrm{O}_{2}$ molecules in the course of the simulation. Dashed lines show the results from GEANT4-DNA converted to numbers of molecules in our simulated conditions [35]. As was discussed above, we cannot directly compare our results with GEANT4-DNA simulations, since in this code the $\mathrm{OH}$ radicals disapear 
and appear through different reaction channels not yet implemented in MBN Explorer. However, the only channel considered there involving $\mathrm{H}_{2} \mathrm{O}_{2}$ is reaction (7). Thus, we can directly compare the evolution of the number of $\mathrm{H}_{2} \mathrm{O}_{2}$ molecules, and rescale the GEANT4-DNA results for $\mathrm{OH}$ as if only reaction (7) happened. In such a case, the number of $\mathrm{OH}$ radicals would be given by:

$$
N_{\mathrm{OH}}=N_{\mathrm{OH}, 0}-2 N_{\mathrm{H}_{2} \mathrm{O}_{2}},
$$

where $N_{\mathrm{OH}, 0}$ is the initial number of $\mathrm{OH}$ radicals, $N_{\mathrm{H}_{2} \mathrm{O}_{2}}$ is the number of peroxide molecules and two $\mathrm{OH}$ disapear each time a peroxide molecule is formed. In our case $N_{\mathrm{OH}, 0}=52$ so for sufficiently long times the final number of peroxide molecules would be 26 . In Figure 5c the dotted line for peroxide is the GEANT4-DNA result rescaled to match $N_{\mathrm{H}_{2} \mathrm{O}_{2}}=26$ at the curve maximum, while the dotted line for $\mathrm{OH}$ is calculated by equation (8) from the rescaled result. The histograms (error bars) correspond to the average (standard deviation) of three independent MBN Explorer simulations. The rate of formation of peroxide (and hence the extinction rate of $\mathrm{OH}$ radicals) matches very well with the evolution predicted by GEANT4-DNA. Therefore, we conlcude that the choice of parameters of the reactive force field is reasonable.

Let us turn now our attention to the case of $200 \mathrm{keV} / \mathrm{u}$ carbon ions where the shock waves are substantial. Figure 6 illustrates the effects of a carbon ion-induced shock wave on the $\mathrm{OH}$ chemistry in the Bragg peak region. Panel (a) shows the OH MSD in a simulation where the shock wave is allowed to develop (solid line) and in another one where the shock wave is artificially switched off (dashed line), forcing $v_{i, j}^{\mathrm{MD}}=v_{i, j}^{\mathrm{eq}}$. Here $\mathrm{OH}$ recombination is not included so essentially the diffusion of $\mathrm{OH}$ radicals without a wave is compared to a collective flow transport with the wave. In this case we obtain (for the case where the shock wave is switched off) a diffusion coefficient $D_{\mathrm{OH}}=0.17 \AA^{2} / \mathrm{ps}$, again in a relatively good agreement with the values found in the literature $[50,53,54]$. It should be noted that this value for the diffusion coefficient should be considered similar to that obtained for $500 \mathrm{keV}$ protons $\left(0.22 \AA^{2} / \mathrm{ps}\right)$; the diffusion coefficient is an inherent property of the chemical species in a medium, and the differences found here should only be attributed to statistical differences between one simulation and the other. In the case of the shock wave the MSD also grows linearly with time but the slope is more than 80 times larger, due to the $\mathrm{OH}$ radical transport by the collective flow. Although the solid line in Figure 6a seems to correspond to the diffusive behavior, radicals are transported by the collective flow. The linearity of time dependencies of displacements in both cases is coincidental. The front radius increases proportionally to $\sqrt{t}$ (insets of Fig. 3), as it can be seen from the hydrodynamic equation [12], and so does the square root of the MSD. This result clearly demonstrates the ability of the shock wave to propagate free radicals (and other reactive species) more efficiently.

Finally, Figure $6 \mathrm{~b}$ shows the results for the number of $\mathrm{OH}$ and $\mathrm{H}_{2} \mathrm{O}_{2}$ molecules in the simulations where

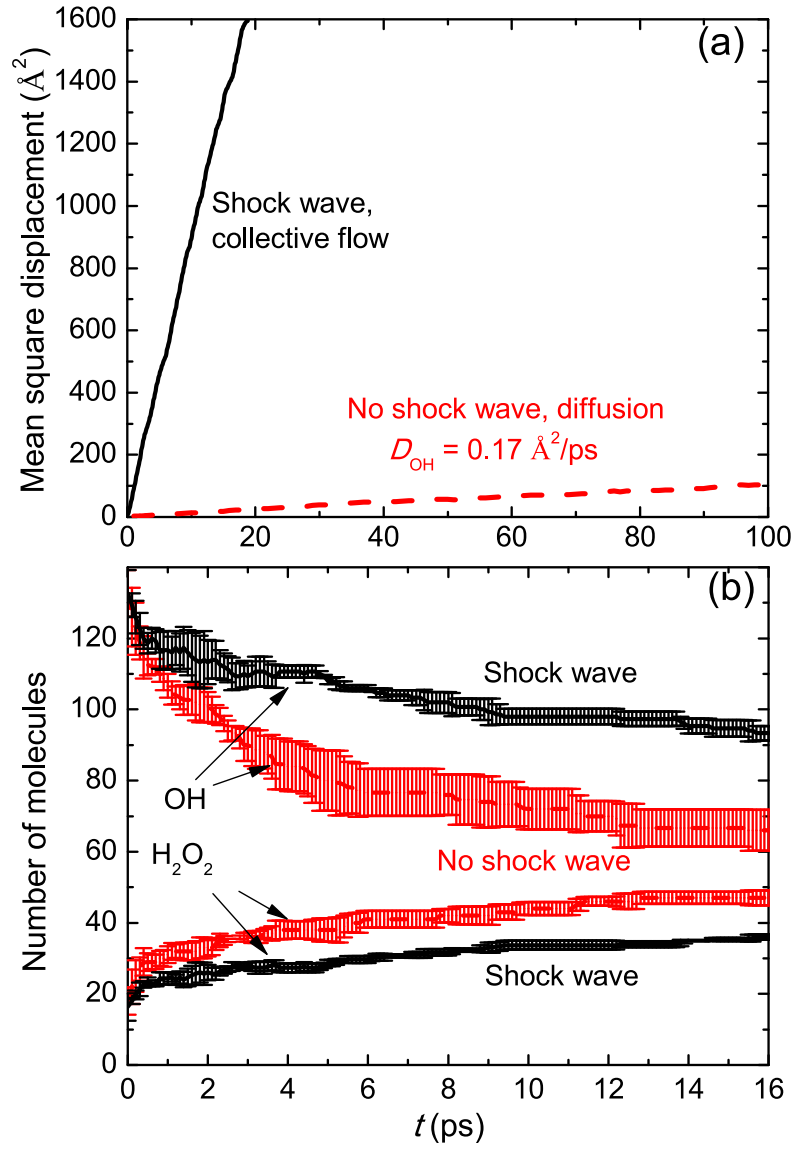

Fig. 6. (a) Mean square displacement of the $\mathrm{OH}$ radicals produced around a $200 \mathrm{keV} / \mathrm{u}$ carbon ion path. (b) Time evolution of the number of $\mathrm{OH}$ radicals and produced $\mathrm{H}_{2} \mathrm{O}_{2}$ molecules. Results of simulations where the shock wave is allowed to develop (transport by the colective flow, solid lines) and where it is artificially "switched off" (transport by diffusion, dashed lines) are shown, in order to demonstrate the shock wave effects on the radiation chemistry.

the chemistry has been included, with the shock wave allowed (solid line) and switched off (dashed line). The lines show averages for three independent simulations with the error bars representing standard deviations. The numbers of $\mathrm{OH}$ radicals as a function of time with and without the shock wave are very different. The shock wave prevents $\mathrm{OH}$ recombination, both by spreading out the radicals (as discussed above) and by creating harsh conditions in which the formation of the $\mathrm{O}-\mathrm{O}$ bond is suppressed. Although only short times were simulated here this tendency may prevail over longer times. After $16 \mathrm{ps}$ of transport with a wave the number of surviving $\mathrm{OH}$ radicals is $40 \%$ larger than that propagated by diffusion. Unfortunately, experimental data are so far only reported for relatively low values of LET [35,53,55], and experiments in the Bragg peak region would be needed to compare with our simulations. In any case, present results are in line with the observations made for the only reported data for water radiolysis with very high-LET ions [32], where unusually large amounts of radicals escaped ion tracks. 
The long times at which G-values are reported in most of the experimental studies of water radiolysis are still challenging for molecular dynamics simulations. However G-values can be probed down to some hundreds of picosends [56]. Furthermore, ultrafast measurements using the pump-probe technique using laser-accelerated ions described by Dromey et al. [57,58] could potentially probe the time-dependence of the $\mathrm{OH}$ signal on the timescales presented here, creating an opportunity to detect a chemical signature of the shock waves and directly prove their existence.

It should be noted that, apart from the experimental oportunity to detect the effect of shock waves in the pure water medium, the observed difference in the number of $\mathrm{OH}$ radicals can have important biological consequences: the fewer $\mathrm{OH}$ radicals recombine to form peroxide, the more are available to damage DNA molecules, hence increasing indirect biodamage. In future work, the effect of shock waves can be combined with the simulation of the DNA damage by $\mathrm{OH}[42,43]$ in order to assess indirect damage for high-LET radiation.

\section{Conclusion}

In this work we have investigated the strength of carboninduced shock waves inside and outside of the Bragg peak region and assessed their impact on the chemistry around the ion path.

Realistic radial doses around ion paths have been calculated by means of the diffusion equations for low energy electrons, where the contribution of $\delta$-electrons has been incorporated, showing a good agreement with Monte Carlo simulation results. The calculated radial doses have been used as the initial conditions for classical molecular dynamics which allows us to simulate ion induced shock waves both in and out of the Bragg peak region. It has been shown that the production of energetic $\delta$-electrons outside of the Bragg peak region substantially weakens the shock waves, this phenomenon being then confined to the Bragg peak.

The new reactive CHARMM force field implemented in MBN Explorer has been used to study the first moments of the induced radiation chemistry in the presence of the shock waves in the form of one of the main cellular reactions, $\mathrm{OH}$ recombination to give hydrogen peroxide. The validity of the reactive force field has been checked by comparison with GEANT4-DNA simulation results for low LET protons, where shock waves do not play an important role. We have found good agreement with Monte Carlo simulations and we predict a diffusion coeffitient for $\mathrm{OH}$ radicals $\left(0.17-0.22 \AA^{2} / \mathrm{ps}\right)$ in reasonable agreement with the value used in Monte Carlo simulations [53,54] and predicted from recent atomistic simulations [50].

The collective flow initiated by the shock wave for carbon ions in the Bragg peak region propagates the radicals 80 times faster than diffusion which is the only means for transport of radicals in the absence of shock waves. The waves also prevent $\mathrm{OH}$ recombination producing hydrogen peroxide. This demonstrates how the shock waves can not only produce direct physical effects (such as rupture of
DNA molecules by high pressure stresses) but also modify the chemical stage of radiation damage. This fact, apart from implying a strong overlap of the physical and chemical stages, also suggests an indirect way for experimentally detecting the ion-induced shock waves, thus far only predicted theoretically. The presented results seem to go in the same direction as the observations made in the only reported water radiolysis experiment at very high-LET [32], where unusually large amounts of radicals escaped ion tracks. In any case, the current developments on picosecond pulsed laser-accelerated ion sources $[57,58]$ will soon provide the possibility to experimentally verify the present results.

The chemical effect has been exemplified here only by the main radiation chemistry reaction of $\mathrm{OH}$ recombination for simplicity. However, further development of the reactive force field in MBN Explorer, where other reactions can be included and/or water dissociation by the shock wave accounted for, may reveal further influence of the shock waves on the chemical stage of radiation. In future work, the simulation of the shock wave effects on the induced radiation chemistry might be combined with a detailed simulation of DNA damage by free radicals, in order to approriately include indirect biodamage within the multiscale approach for the radiation damage produced by ion beams.

The authors recognize financial support from the European Union's FP7-People Program within the Initial Training Network No. 608163 "ARGENT". PdV acknowledges additional support from the Sir John Mason Academic Trust with a small travel grant.

\section{Author contribution statment}

The radial dose calculations and reactive molecular dynamics simulations were performed by PdV under the supervision of AVS and ES. The manuscript was mainly drafted by $\mathrm{PdV}$, following discussions with AVS, ES, FJC and NJM. All authors contributed to the conceptual design of the work, actively participated in the discussion of the results, provided valuable comments and insight, and contributed to the revision of the manuscript.

Open Access This is an open access article distributed under the terms of the Creative Commons Attribution License (http://creativecommons.org/licenses/by/4.0), which permits unrestricted use, distribution, and reproduction in any medium, provided the original work is properly cited.

\section{References}

1. F.A. Cucinotta, M. Durante, Lancet Oncol. 7, 431 (2006)

2. D. Schardt, T. Elsässer, D. Schulz-Ertner, Rev. Mod. Phys. 82, 383 (2010)

3. J.S. Loeffler, M. Durante, Nat. Rev. Clin. Oncol. 10, 411 (2013)

4. E. Surdutovich, A.V. Solov'yov, Eur. Phys. J. D 68, 353 (2014) 
5. A.V. Solov'yov (Ed.), Nanoscale Insights into Ion-Beam Cancer Therapy (Springer Int. Pub., Switzerland, 2017)

6. E.L. Alpen, Radiation Biophysics (Academic Press, 1998)

7. R. Hawkins, Int. J. Radiat. Biol. 69, 739 (1996)

8. N. Scholz, G. Kraft, Adv. Space Res. 18, 5 (1996)

9. R. Stewart et al., Phys. Med. Biol. 60, 8249 (2015)

10. W. Friedland et al., Sci. Rep. 7, 45161 (2017)

11. A.V. Solov'yov, E. Surdutovich, E. Scifoni, I. Mishustin, W. Greiner, Phys. Rev. E 79, 011909 (2009)

12. E. Surdutovich, A.V. Solov'yov, Phys. Rev. E 82, 051915 (2010)

13. A.V. Verkhovtsev, E. Surdutovich, A.V. Solov'yov, Sci. Rep. 6, 27654 (2016)

14. P. de Vera, R. Garcia-Molina, I. Abril, A.V. Solov'yov, Phys. Rev. Lett. 110, 148104 (2013)

15. P. de Vera, I. Abril, R. Garcia-Molina, A.V. Solov'yov, J. Phys.: Conf. Ser. 438, 012015 (2013)

16. E. Surdutovich, A.V. Solov'yov, Eur. Phys. J. D 69, 193 (2015)

17. L. Landau, E. Lifshitz, in Fluid Dynamics, 2nd edn. (Reed-Elsevier, Oxford, Boston, Johannesburg, 1987), Vol. 6

18. Y. Zel'dovich, Y. Raiser, in Physics of Shock Waves and High- Temperature Hydrodynamic Phenomena (Oxford Press, New York, 1966), Vol. 1

19. Y.B. Zel'dovich, J. Exp. Theor. Phys. 12, 389 (1942)

20. H.A. Bethe et al., Blast wave, Los Alamos Report LA2000, Ch. 2, Los Alamos, 1947

21. G.I. Taylor, Proc. Roy. Soc. Lond. A201, 159 (1950)

22. L.I. Sedov, Prokl. Mat. Mek. 10, 241 (1946)

23. J. von Neumann, The point source solution, in Blast wave, edited by K. Fuchs, J.O. Hirschfelder, J.L. Magee, R. Peierls, J. von Neumann (Los Alamos, 1947)

24. P. Krehl, History of shock waves, in Handbook of shock waves, edited by G. Ben-Dor, O. Igra, T. Elperin (Academic Press, San Diego, 2001), Vol. 1

25. P.O.K. Krehl, History of shock waves, explosions and impact, in $A$ chronological and biographical reference (Springer-Verlag, Berlin, Heidelberg, 2009)

26. A.V. Yakubovich, E. Surdutovich, A.V. Solov'yov, AIP Conf. Proc. 1344, 230 (2011)

27. P. de Vera, N.J. Mason, F.J. Currell, A.V. Solov'yov, Eur. Phys. J. D 70, 183 (2016)

28. P. de Vera, E. Surdutovich, N.J. Mason, A.V. Solov'yov, Eur. Phys. J. D 71, 281 (2017)

29. A. Mozumder, Y. Hatano, Charged Particle and Photon Interaction with Matter: Chemical, Physicochemical and Biological Consequences with Applications (Marcel Dekker Inc., New York, 2004)

30. E. Surdutovich, A.V. Yakubovich, A.V. Solov'yov, Sci. Rep. 3, 1289 (2013)

31. N. Baily, Med. Phys. 19, 525 (1992)

32. J. A. LaVerne, R.H. Schuler, J. Phys. Chem. 100, 16034 (1996)
33. I.A. Solov'yov, A.V. Yakubovich, P.V. Nikolaev, I. Volkovets, A.V. Solov'yov, J. Comp. Chem. 33, 2412 (2012), available at: www.mbnexplorer.com

34. G.B. Sushko, I.A. Solov'yov, A.V. Verkhovtsev, S.N. Volkov, A.V. Solov'yov, Eur. Phys. J. D 70, 12 (2016)

35. M. Karamitros, S. Luan, M.A. Bernal, J. Allison, G. Baldacchino, M. Davidkova, Z. Francis, W. Friedland, V. Ivantchenko, A. Ivantchenko, A. Mantero, P. Nieminem, G. Santin, H.N. Tran, V. Stepan, S. Incerti, J. Comput. Phys. 274, 841 (2014)

36. M.A. Xapsos, Rad. Res. 132, 282 (1992)

37. L.G. Gerchikov, A.N. Ipatov, A.V. Solov'yov, W. Greiner, J. Phys. B 33, 4905 (2000)

38. M.P.R. Waligórski, R.N. Hamm, R. Katz, Int. J. Rad. Appl. Instrum. 11, 309 (1986)

39. T. Liamsuwan, H. Nikjoo, Phys. Med. Biol. 58, 673 (2013)

40. S. Incerti et al., Nucl. Instr. Meth. B 333, 92 (2014)

41. M. Toulemonde, E. Surdutovich, A.V. Solov'yov, Phys. Rev. E 80, 031913 (2009)

42. R.M. Abolfath, A.C.T. van Duin, T. Brabec, J. Phys. Chem. A 115, 11045 (2011)

43. R.M. Abolfath, D.J Carlson, Z.J. Chen, R. Nath, Phys. Med. Biol. 58, 7143 (2013)

44. M.P. Allen, D.J. Tildesley, Computer simulation of liquids (Oxford University Press, Oxford, 1989)

45. A.D. MacKerell, Jr. et al., J. Phys. Chem. B 102, 3586 (1998)

46. T. Douki, J. Onuki, M.H.G. Medeiros, E.J. Bechara, J. Cadet, P. Di Mascio, FEBS Lett. 428, 93 (1998)

47. C. von Sonntag, The Chemical Basis of Radiation Biology (Taylor \& Francis, London, 1987)

48. B. Ruscic et al., J. Phys. Chem. A 106, 2727 (2002)

49. S.J. Blanksby, G. Barney Ellison, Acc. Chem. Res. 36, 255 (2003)

50. A. Pabis, J. Szala-Bilnik, D. Swiatla-Wojcik, Phys. Chem. Chem. Phys. 13, 9458 (2011)

51. L. De Gioia, P. Fantucci, J. Mol. Struct. 469, 41 (1999)

52. F. Agapito, B.J. Costa Cabral, J.A. Martinho Simoes, J. Mol. Struct. 729, 223 (2005)

53. M.S. Kreipl, W. Friedland, H.G. Paretzke, Radiat. Environ. Biophys. 48, 11 (2009)

54. M. Karamitros et al., Progr. Nucl. Sci. Tech. 2, 503 (2011)

55. T. Maeyama, S. Yamashita, G. Baldacchino, M. Taguchi, A. Kimura, T. Murakami, Y. Katsumura, Rad. Phys. Chem. 80, 1352 (2011)

56. C.D. Jonah, J.R. Miller, J. Phys. Chem. 81, 1974 (1977)

57. B. Dromey, M. Coughlan, L. Senje, M. Taylor, S. Kuschel, B. Villagomez-Bernabe, R. Stefanuik, G. Nersisyan, L. Stella, J. Kohanoff, M. Borghesi, F. Currell, D. Riley, D. Jung, C.-G. Wahlström, C.L.S. Lewis, M. Zepf, Nat. Commun. 7, 10642 (2016)

58. L. Senje, M. Coughlan, D. Jung, M. Taylor, G. Nersisyan, D. Riley, C.L.S. Lewis, O. Lundh, C.-G. Wahlström, M. Zepf, B. Dromey, Appl. Phys. Lett. 110, 104102 (2017) 